\title{
Mobile communication traffic analysis on a road systems model
}

K. Nakano, K. Saita, M. Sengoku

Department of Information Engineering, Niigata University

2-8050, Ikarashi, Niigata 950-2181, Japan

Telephone/Telefax: +8125 262 7219

E-mail: nakano@info.eng.niigata-u.ac.jp

URL: http://www.info.eng.niigata-u.ac.jp/tiger/

\section{Y. Yamada}

YRP Mobile Telecommunications Key Technology Research

Laboratories Co., Ltd.

3-4, Hikari-no-oka, Yokosuka 239-0847, Japan

\section{S. Shinoda}

Department of Electrical and Electronic Engineering

Chuo University

1-13-27, Kasuga, Bunkyo-ku, Tokyo 112-8551, Japan

\begin{abstract}
This paper describes communication traffic characteristics of a cellular mobile communication system on a road network assuming that calls are generated by subscribers on vehicles moving along the road network. The offered load and the blocking probability are analyzed. For this analysis, the hand-off probability and the mean channel occupancy time are also analyzed. This paper utilizes the results of survey and estimation of traffic flow on road networks discussed in the transportation engineering field.
\end{abstract}

\section{Keywords}

Mobile communication, cellular system, communication traffic, traffic flow, road system, road network 


\section{INTRODUCTION}

A cellular mobile communication system comprises a lot of radio zones which are called cells. Each cell has a base station. Suppose that a new call arrives at cell $A$. The base station in cell $A$ assigns a communication channel to this new call. The call communicates to the base station in cell $A$. If the call leaves cell $A$ and enters cell $B$, which is adjacent to cell $A$, the active call releases the channel of cell $A$ and the base station in cell $B$ assigns a communication channel to the active call. This operation is called a hand-off operation. The call, which enters cell $B$, is called a hand-off call. An active call crossing a cell boundary always requires a hand-off operation. Of course, in the hand-off operation, if cell $B$ has no available channel, the hand-off call is blocked.

The probability that a call requires a hand-off operation is called the handoff probability. The channel occupancy time is the time during which an active call holds a channel in a cell. Because mobility of subscribers to mobile communication systems causes the hand-off operations, mobility of subscribers influences the hand-off probability and the channel occupancy time. Also, new calls and hand-off calls arrive at a group of channels of a cell, and the calls hold only during their channel occupancy times. Therefore, mobility of the subscribers also influences the offered load. Hence, analyzing the communication traffic characteristics of the cellular systems, we should consider the influence of mobility of the subscribers.

There exist some important results on analysis of relation between mobility of subscribers to cellular systems and communication traffic characteristics of cellular systems. A technique to analyze the hand-off probability, the channel occupancy time and the blocking probability in the 1-dimensional cellular system, e.g. the high way cellular system, has been proposed (Otsuka,1987). In this case, it is assumed that subscribers move along only one route. However, subscribers in the 2-dimensional cellular system on a road network, which lies on the 2-dimensional plane, move along various kinds of routes on the road network. Furthermore, the volume of the traffic flow on a route is different from these of other routes. Intuitively, it is considered that this difference causes spatially heterogeneous communication traffic characteristics of cellular systems. We should consider the influence of the difference of the volume of the traffic flow on each route of the road network when we analyze communication traffic characteristics of the 2-dimensional cellular system. As related works, some techniques have been proposed (Guerin,1987) (Hong,1986) (Inoue,1994) (Nanda,1993) (Rappaport,1991) (Rappaport,1994); however, in these articles, structure of road networks and the volume of the traffic flow on each route of the road networks are not considered.

With these points as background, we analyze the communication traffic characteristics of the 2-dimensional cellular mobile communication systems. In this analysis, we are given a road network and a cellular system which lies 


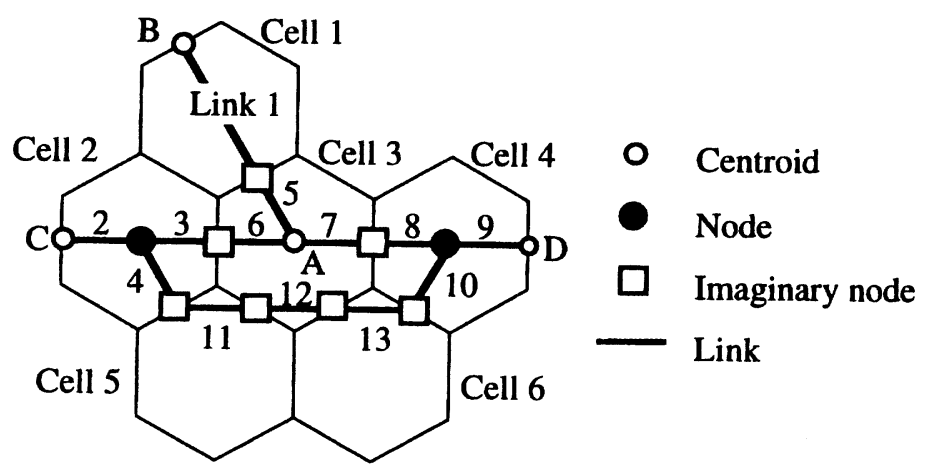

Figure 1 An example.

on the road network. We are also given the traffic volume on each route on the road network, the velocity of vehicles and the length of each street. These values can be estimated by results reported in the transportation engineering field. Our problems are to analyze the blocking probability in each cell of the cellular system.

Section 2 gives some definitions and assumptions for the analysis. Section 2 also describes a method to analyze the communication traffic characteristics. Section 3 describes a method to estimate traffic volume on each route of a road network and velocity of vehicles. Section 4 gives numerical results. Section 5 concludes this paper.

\section{ANALYSIS}

\subsection{Definitions and assumptions}

In the following, we make definitions and assumptions:

(A1) Suppose that a cellular system comprises cells which are modeled by regular hexagons and that the cellular system lies on a road system. This road system is modeled by a road network which consists of nodes and links which correspond to intersections and streets, respectively. We introduce imaginary nodes to represent cell boundaries on the road network. Figure 1 shows relation between a cellular system and a road network. As shown in Figure 1, there exists an imaginary node on an intersection of a cell boundary and a link. Also we introduce centroids which are special nodes to represent the origin and the destination of the traffic flow. Centroids are also shown in Figure 1. 


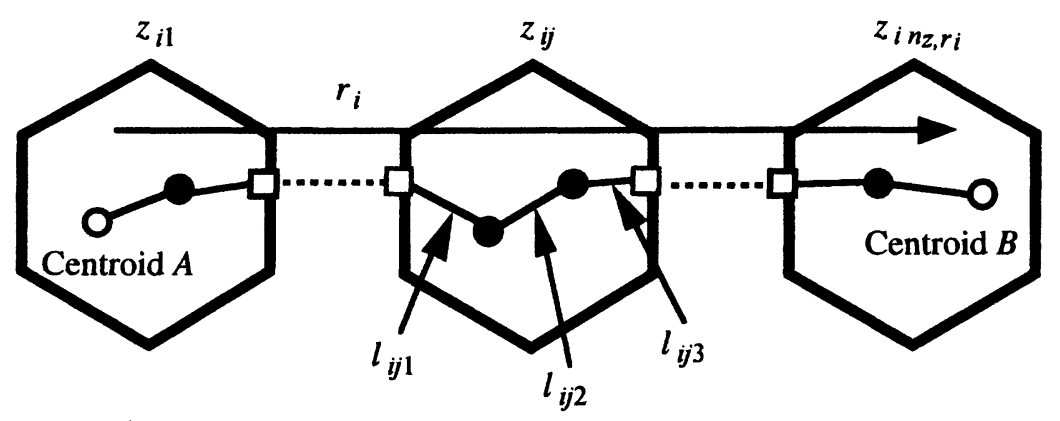

Figure 2 Relation between $r_{i}, z_{i j}$ and $l_{i j k}$.

(A2) Suppose that each vehicle starts its travel at a centroid and finishes its travel at another centroid. We define that a route is a path from a centroid to another centroid. We distinguish between a route from centroid $A$ to centroid $B$ and a route from centroid $B$ to centroid $A$ even if these routes comprise the same set of links. Let $n_{r}$ be the number of available routes in the system. Let $r_{i}$ be route $i$, where $i=1, \ldots, n_{r}$. In this paper, a vehicle flowing along route $r$ means a vehicle which starts at the origin of route $r$ and finishes its trip at the destination of route $r$ after flowing along route $r$. A vehicle moving along route $r$ has the same meaning. Consider vehicles flowing along $r_{i}$. Suppose that $r_{i}$ is from centroid $A$ to centroid $B$. These vehicles originate at centroid $A$, go through some cells and arrive at centroid $B$, which is the end of $r_{i}$. We define that $z_{i j}$ is the $j$ th cell which the vehicles go through. Let $n_{z, r_{i}}$ be the number of the cells on $r_{i}$. The vehicles must enter $z_{i j}$. We define that $l_{i j k}$ is the $k$ th link which these vehicles go through after entering $z_{i j}$. Let $n_{l, z_{i j}}$ be the number of the links which are in $z_{i j}$ and are also on $r_{i}$. Figure 2 shows an example of a route. In this case, $n_{l, z_{i j}}=3$.

(A3) Consider vehicles moving along route $r$. Let $Q(r)$ be the number of vehicles which start at the origin of route $r$ and finish their trips at the destination of route $r$ after flowing route $r$ per unit time. $Q(r)$ vehicles which move along route $r$ go through a point on route $r$ for an unit time. $Q(r)$ is called the traffic volume on route $r$. We assume that $Q\left(r_{i}\right)$ is given in advance for all $i$.

(A4) We assume that the distribution of vehicles on a link is uniform.

(A5) We assume that the length of every link is given in advance. Let $d(l)$ be the length of link $l$.

(A6) Consider vehicles moving along $r_{i}$. For simplicity, we assume that these vehicles move with the same velocity on a link. Let $v\left(l_{i j k}\right)$ be the velocity of the vehicles, which move along $r_{i}$, on $l_{i j k}$. 
(A7) Consider $Q\left(r_{i}\right)$ and $v\left(l_{i j k}\right)$. We assume that $Q\left(r_{i}\right)=K\left(l_{i j k}\right) v\left(l_{i j k}\right)$, where $K\left(l_{i j k}\right)$ is the traffic density on $l_{i j k}$, which means the number of vehicles flowing along $r_{i}$ per unit length on $l_{i j k}$. This relation is derived in the transportation engineering field (Takeuchi,1986).

(A8) We assume that call arrivals of new calls and those of hand-off calls form Poisson processes, respectively. Considering new call arrivals from $p$ vehicles, we assume that the call originating rate of the new calls is proportional to $p$ and is equal to $\lambda p$, where $\lambda$ is a constant value. We assume that the number of vehicles in a cell is sufficiently larger than that of channels.

(A9) We assume that the holding time of a call, which means the life of the call, is an exponential random variable with a mean of $h_{0}$.

(A10) We assume that every cell has the same number of channels. Let $S$ be the number of channels in a cell.

(A11) We assume that the velocities of vehicles on the links connected by an imaginary node are the same.

\subsection{Offered load and blocking probability}

Consider new calls which originate in cell $z$. Suppose that these new calls are generated by subscribers on vehicles moving along route $r$. Let $\lambda_{n c}(r, z)$ be the call originating rate of the new calls in cell $z$. Let $h_{n c}(r, z)$ be the mean value of the channel occupancy times during which the new calls hold channels in cell $z$. Consider a vehicle moving along $r_{i}$. Suppose that this vehicle generates a new call in $z_{i j}$. Let $P_{H, n c}\left(r_{i}, z_{i j}\right)$ be the probability that the new call is accepted in $z_{i j}$ and requires a hand-off operation in $z_{i j+1}$. Consider hand-off calls which enter cell $z$. Suppose that these hand-off calls move along route $r$. Let $\lambda_{h c}(r, z)$ be the call originating rate of the hand-off calls in cell $z$. Let $h_{h c}(r, z)$ be the mean value of the channel occupancy times during which the hand-off calls hold channels in cell $z$. Consider an active call moving along $r_{i}$. Suppose that this call requires a hand-off operation in $z_{i j}$. Let $P_{H, h c}\left(r_{i}, z_{i j}\right)$ be the probability that the call is accepted in $z_{i j}$ and requires a hand-off operation in $z_{i j+1}$.

In a cellular mobile communication system, both new calls and hand-off calls arrive at the group of channels in a cell, and each of the calls holds a channel only during its channel occupancy time, which is shorter than the call's life. If some kinds of Poisson calls arrive at a group of channels, the blocking probability can be calculated by substituting the sum of the offered loads of the calls into the Erlang B formula (Cooper,1981). In (A8), we assume that new calls and hand-off calls are Poisson calls, respectively. We distinguish between the mean channel occupancy time of new calls and that of hand-off calls as defined above. In (A10), we assume that the number of channels is constant in a cell. New calls and hand-off calls in cell $z$ are generated 
by subscribers on vehicles moving along all routes which go through cell $z$. Therefore, the blocking probability in cell $z$, which is denoted by $B(z)$, is represented by the Erlang B formula with $a(z)$, which means the sum of the offered loads of new calls and those of hand-off calls on cell $z$, as follows:

$B(z)=\frac{\frac{a(z)^{S}}{S !}}{\sum_{r=0}^{S} \frac{a(z)^{r}}{r !}}$,

where

$a(z)=\sum_{i=1}^{n_{r}}\left[\lambda_{n c}\left(r_{i}, z\right) h_{n c}\left(r_{i}, z\right)+\lambda_{h c}\left(r_{i}, z\right) h_{h c}\left(r_{i}, z\right)\right]$

where $\lambda_{n c}\left(r_{i}, z\right)=0, \lambda_{h c}\left(r_{i}, z\right)=0, h_{n c}\left(r_{i}, z\right)=0$ and $h_{h c}\left(r_{i}, z\right)=0$ if $r_{i}$ does not go through cell $z$. From (A3)-(A8), $\lambda_{n c}\left(r_{i}, z_{i j}\right)$ is represented as follows:

$\lambda_{n c}\left(r_{i}, z_{i j}\right)=\sum_{k=1}^{n_{l, z_{i j}}} \frac{\lambda Q\left(r_{i}\right) d\left(l_{i j k}\right)}{v\left(l_{i j k}\right)}$.

We can substitute (3) into (2) if $z_{i j}=z . \lambda_{h c}\left(r_{i}, z_{i j}\right)$ is represented as follows:

$$
\begin{aligned}
\lambda_{h c}\left(r_{i}, z_{i j}\right)= & \lambda_{n c}\left(r_{i}, z_{i j-1}\right) P_{H, n c}\left(r_{i}, z_{i j-1}\right) \\
& +\lambda_{h c}\left(r_{i}, z_{i j-1}\right) P_{H, h c}\left(r_{i}, z_{i j-1}\right),
\end{aligned}
$$

where $\lambda_{h c}\left(r_{i}, z_{i 1}\right)=0$ because all vehicles moving along $r_{i}$ start at $z_{i 1}$. We can substitute (4) into (2) if $z_{i j}=z$. In the following sections, we must consider $h_{n c}\left(r_{i}, z\right)$ and $h_{h c}\left(r_{i}, z\right)$ in (2) to define $a(z)$. Also, we must consider $P_{H, n c}\left(r_{i}, z_{i j-1}\right)$ and $P_{H, h c}\left(r_{i}, z_{i j-1}\right)$ in (4) to define $\lambda_{h c}\left(r_{i}, z_{i j}\right)$. As described later, $P_{H, n c}\left(r_{i}, z_{i j-1}\right)$ and $P_{H, h c}\left(r_{i}, z_{i j-1}\right)$ are functions of the blocking probabilities in cells, respectively; then, we can define $a(z)$ as a function of blocking probabilities. As described later, we can define nonlinear simultaneous equations which consist of (1) and (2) of all cells, where variables are only blocking probabilities in cells.

\subsection{Hand-off probability}

We consider $P_{H, n c}\left(r_{i}, z_{i j}\right)$. Consider a vehicle moving along $r_{i}$. Suppose that this vehicle is on $l_{i j k}$ and moves toward $l_{i j k+1}$. Suppose that the vehicle generates a new call on $l_{i j k}$ at a point denoted by $q$. The distance from $q$ to the boundary between $l_{i j k}$ and $l_{i j k+1}$ is denoted by $X . X$ is a random variable. 
From (A4), $\operatorname{Pr}(X \leq x)$, which means the probability that $X \leq x$, corresponds to the ratio of $x$ to $d\left(l_{i j k}\right)$. Then

$\operatorname{Pr}(X \leq x)=\frac{x}{d\left(l_{i j k}\right)}$

Let $T$ be the random variable of the time required to the trip from $q$ to the boundary between $l_{i j k}$ and $l_{i j k+1}$. Let $\operatorname{Pr}(T \leq t)$ be the probability that $T \leq t$. Then

$\operatorname{Pr}(T \leq t)=\frac{v\left(l_{i j k}\right) t}{d\left(l_{i j k}\right)}$

Let $p\left(l_{i j k}, t\right)$ be the probability density function of $(6)$ of $l_{i j k}$, then

$p\left(l_{i j k}, t\right)=\frac{v\left(l_{i j k}\right)}{d\left(l_{i j k}\right)}$

Consider again the new call which is generated at $q$. Suppose that this call is not blocked in $z_{i j}$. The time required for the trip from the boundary between $l_{i j k}$ and $l_{i j k+1}$ to the boundary between $z_{i j}$ and $z_{i j+1}$ is $\sum_{m=k+1}^{n_{l, z_{i j}}} \frac{d\left(l_{i j m}\right)}{v\left(l_{i j m}\right)}$. Let $H$ be the random variable which means the holding time of the call, namely the life of the call. Then, $\operatorname{Pr}\left[H>t+\sum_{m=k+1}^{n_{l, z_{i j}}} \frac{d\left(l_{i j m}\right)}{v\left(l_{i j m}\right)}\right]$ is the probability that the call keeps holding a channel until this call arrives at the cell boundary. As assumed in (A9), the holding time obeys the exponential distribution. Then

$\operatorname{Pr}\left[H>t+\sum_{m=k+1}^{n_{l, z_{i j}}} \frac{d\left(l_{i j m}\right)}{v\left(l_{i j m}\right)}\right]=\exp \left[-\frac{t+\sum_{m=k+1}^{n_{l, z_{i j}}} \frac{d\left(l_{i j m}\right)}{v\left(l_{i j m}\right)}}{h_{0}}\right]$

Consider a vehicle moving along $r_{i}$. Suppose that this vehicle generates a new call on $l_{i j k}$. Let $P_{H, n c}^{\prime}\left(r_{i}, z_{i j}, l_{i j k}\right)$ be the probability that this new call is not blocked in $z_{i j}$ and requires a hand-off operation in $z_{i j+1}$. From (7) and (8), $P_{H, n c}^{\prime}\left(r_{i}, z_{i j}, l_{i j k}\right)$ is represented as follows:

$$
\begin{aligned}
P_{H, n c}^{\prime}\left(r_{i}, z_{i j}, l_{i j k}\right)= & {\left[1-B\left(z_{i j}\right)\right] } \\
& \times \int_{0}^{\frac{d\left(l_{i j k}\right)}{v\left(l_{i j k}\right)}} \operatorname{Pr}\left[H>t+\sum_{m=k+1}^{n_{l, z_{i j}}} \frac{d\left(l_{i j m}\right)}{v\left(l_{i j m}\right)}\right] p\left(l_{i j k}, t\right) d t \\
= & {\left[1-B\left(z_{i j}\right)\right] }
\end{aligned}
$$




$$
\begin{aligned}
& \quad \times \int_{0}^{\frac{d\left(l_{i j k}\right)}{v\left(l_{i j k}\right)}} \exp \left[-\frac{t+\sum_{m=k+1}^{n_{l, z_{i j}}} \frac{d\left(l_{i j m}\right)}{v\left(l_{i j m}\right)}}{h_{0}}\right] \frac{v\left(l_{i j k}\right)}{d\left(l_{i j k}\right)} d t \\
& =\quad\left[1-B\left(z_{i j}\right)\right] \frac{v\left(l_{i j k}\right) h_{0}}{d\left(l_{i j k}\right)} \exp \left[-\frac{\sum_{m=k+1}^{n_{l, z_{i j}}} \frac{d\left(l_{i j m}\right)}{v\left(l_{i j m}\right)}}{h_{0}}\right] \\
& \quad \times\left\{1-\exp \left[-\frac{d\left(l_{i j k}\right)}{v\left(l_{i j k}\right) h_{0}}\right]\right\} .
\end{aligned}
$$

Consider a vehicle moving along $r_{i}$. Suppose that this vehicle generates a new call in $z_{i j}$. Let $P_{l}\left(r_{i}, z_{i j}, l_{i j k}\right)$ be the probability that this new call originates on $l_{i j k}$. Let $\lambda_{n c}^{\prime}(r, z, l)$ be the call originating rate of new calls which are generated by subscribers on vehicles which flow along route $r$, are in cell $z$ and are on link $l$. Then

$P_{l}\left(r_{i}, z_{i j}, l_{i j k}\right)=\frac{\lambda_{n c}^{\prime}\left(r_{i}, z_{i j}, l_{i j k}\right)}{\sum_{m=1}^{n_{l, z_{i j}}} \lambda_{n c}^{\prime}\left(r_{i}, z_{i j}, l_{i j m}\right)}$

From (A3)-(A8), $\lambda_{n c}^{\prime}\left(r_{i}, z_{i j}, l_{i j k}\right)$ is represented as follows:

$$
\lambda_{n c}^{\prime}\left(r_{i}, z_{i j}, l_{i j k}\right)=\frac{\lambda Q\left(r_{i}\right) d\left(l_{i j k}\right)}{v\left(l_{i j k}\right)}
$$

From (10) and (11),

$$
P_{l}\left(r_{i}, z_{i j}, l_{i j k}\right)=\frac{\frac{\lambda Q\left(r_{i}\right) d\left(l_{i j k}\right)}{v\left(l_{i j k}\right)}}{\sum_{m=1}^{n_{l, z_{i j}}} \frac{\lambda Q\left(r_{i}\right) d\left(l_{i j m}\right)}{v\left(l_{i j m}\right)}}=\frac{\frac{d\left(l_{i j k}\right)}{v\left(l_{i j k}\right)}}{\sum_{m=1}^{n_{l, z_{i j}} \frac{d\left(l_{i j m}\right)}{v\left(l_{i j m}\right)}}}
$$

From (9) and (12),

$$
\begin{aligned}
P_{H, n c}\left(r_{i}, z_{i j}\right)= & \sum_{k=1}^{n_{l, z_{i j}}} P_{H, n c}^{\prime}\left(r_{i}, z_{i j}, l_{i j k}\right) P_{l}\left(r_{i}, z_{i j}, l_{i j k}\right) \\
= & {\left[1-B\left(z_{i j}\right)\right] \frac{h_{0}}{\sum_{m=1}^{n_{l, z_{i j}} \frac{d\left(l_{i j m}\right)}{v\left(l_{i j m}\right)}}} } \\
& \times \sum_{k=1}^{n_{l, z_{i j}}} \exp \left[-\frac{\sum_{m=k+1}^{n_{l, z_{i j}}} \frac{d\left(l_{i j m}\right)}{v\left(l_{i j m}\right)}}{h_{0}}\right] \\
& \times\left\{1-\exp \left[-\frac{d\left(l_{i j k}\right)}{v\left(l_{i j k}\right) h_{0}}\right]\right\}
\end{aligned}
$$


where $j<n_{z, r_{i}}$. From (13), we can define $P_{H, n c}\left(r_{i}, z_{i j}\right)$ as a function of $B\left(z_{i j}\right)$.

Next, we consider a hand-off call which enters $z_{i j}$ along $r_{i} . P_{H, h c}\left(r_{i}, z_{i j}\right)$ is the probability that this call is not blocked in $z_{i j}$ and again hand-offs to $z_{i j+1}$. In this case, we can consider that the hand-off call originates at the cell boundary between $z_{i j-1}$ and $z_{i j}$, then the travel time to $z_{i j+1}$ is equal to $\sum_{m=1}^{n_{l, z_{i j}}} \frac{d\left(l_{i j m}\right)}{v\left(l_{i j m}\right)}$. The holding time has the memoryless property because the holding time obeys the exponential distribution. Therefore, from (8), $P_{H, h c}\left(r_{i}, z_{i j}\right)$ is represented as follows:

$$
\begin{aligned}
P_{H, h c}\left(r_{i}, z_{i j}\right) & =\left[1-B\left(z_{i j}\right)\right] \operatorname{Pr}\left[H>\sum_{m=1}^{n_{l, z_{i j}}} \frac{d\left(l_{i j m}\right)}{v\left(l_{i j m}\right)}\right] \\
& =\left[1-B\left(z_{i j}\right)\right] \exp \left[-\frac{\sum_{m=1}^{n_{l, z_{i j}}} \frac{d\left(l_{i j m}\right)}{v\left(l_{i j m}\right)}}{h_{0}}\right],
\end{aligned}
$$

where $j<n_{z, r_{i}}$.

$z_{i n_{z, r_{i}}}$ is the destination of vehicles moving along $r_{i}$. Therefore, calls in $z_{i n_{z, r_{i}}}$ request no hand-off operation. Then

$P_{H, n c}\left(r_{i}, z_{i n_{z, r_{i}}}\right)=0$

$P_{H, h c}\left(r_{i}, z_{i n_{z, r_{i}}}\right)=0$.

By substituting (13)-(16) into (4), we can define $\lambda_{h c}\left(r_{i}, z_{i j}\right)$ as a function of the blocking probabilities in cells.

\subsection{Channel occupancy time}

Consider vehicles moving along $r_{i}$. Consider calls which are generated by subscribers on these vehicles. Suppose that these calls originate on $l_{i j k}$ at a point denoted by $q$. Suppose that these calls are not blocked in $z_{i j}$. Suppose that the travel time of each of these calls from $q$ to the boundary between $l_{i j k}$ and $l_{i j k+1}$ is $t$. Then, the travel time of each of these calls from $q$ to the boundary between $z_{i j}$ and $z_{i j+1}$ is $t+\sum_{m=k+1}^{n_{l, z_{i j}}} \frac{d\left(l_{i j m}\right)}{v\left(l_{i j m}\right)}$. Suppose that the life of one of these calls is equal to $\tau$. Then, if $\tau$ is greater than or equal to $t+\sum_{m=k+1}^{n_{l, z_{i j}}} \frac{d\left(l_{i j m}\right)}{v\left(l_{i j m}\right)}$, the channel occupancy time of this call is equal to $t+\sum_{m=k+1}^{n_{l, z_{i j}}} \frac{d\left(l_{i j m}\right)}{v\left(l_{i j m}\right)}$. If $\tau$ is smaller than $t+\sum_{m=k+1}^{n_{l, z_{i j}}} \frac{d\left(l_{i j m}\right)}{v\left(l_{i j m}\right)}$, then the channel occupancy time of this call is equal to $\tau$. The holding time obeys the exponential distribution. Therefore, $h_{n c}^{\prime \prime}\left(r_{i}, z_{i j}, l_{i j k}, t\right)$, which means the mean channel occupancy time of the calls 
which originate at $q$, is represented as follows:

$$
\begin{aligned}
h_{n c}^{\prime \prime}\left(r_{i}, z_{i j}, l_{i j k}, t\right)= & \int_{0}^{t+\sum_{m=k+1}^{n_{l, z_{i j}}} \frac{d\left(l_{i j m}\right)}{v\left(l_{i j m}\right)}} \frac{\tau}{h_{0}} \exp \left(-\frac{\tau}{h_{0}}\right) d \tau \\
& +\int_{t+\sum_{m=k+1}^{n_{l, z_{i j}}} \frac{d\left(l_{i j m}\right)}{v\left(l_{i j m}\right)}}^{\infty}\left[t+\sum_{m=k+1}^{n_{l, z_{i j}}} \frac{d\left(l_{i j m}\right)}{v\left(l_{i j m}\right)}\right] \\
& \times \frac{1}{h_{0}} \exp \left(-\frac{\tau}{h_{0}}\right) d \tau \\
= & h_{0}\left\{1-\exp \left[-\frac{t+\sum_{m=k+1}^{n_{l, z_{i j}}} \frac{d\left(l_{i j m}\right)}{v\left(l_{i j m}\right)}}{h_{0}}\right]\right\} .
\end{aligned}
$$

Consider vehicles moving along $r_{i}$. Consider calls which are generated on $l_{i j k}$ by subscribers on these vehicles. Suppose that these calls are not blocked in $z_{i j}$. Let $h_{n c}^{\prime}\left(r_{i}, z_{i j}, l_{i j k}\right)$ be the mean value of channel occupancy times of these calls. From (7) and (17),

$$
\begin{aligned}
h_{n c}^{\prime}\left(r_{i}, z_{i j}, l_{i j k}\right)= & \int_{0}^{\frac{d\left(l_{i j k}\right)}{v\left(l_{i j k}\right)}} h_{n c}^{\prime \prime}\left(r_{i}, z_{i j}, l_{i j k}, t\right) p\left(l_{i j k}, t\right) d t \\
= & \int_{0}^{\frac{d\left(l_{i j k}\right)}{v\left(l_{i j k}\right)}} h_{0}\left\{1-\exp \left[-\frac{\left.\left.t+\sum_{m=k+1}^{n_{l, z_{i j}} \frac{d\left(l_{i j m}\right)}{v\left(l_{i j m}\right)}}\right]\right\}}{h_{0}}\right]\right\} \\
& \times \frac{v\left(l_{i j k}\right)}{d\left(l_{i j k}\right)} d t \\
= & h_{0}-h_{0}{ }^{2} \frac{v\left(l_{i j k}\right)}{d\left(l_{i j k}\right)} \exp \left[-\frac{\sum_{m=k+1}^{n_{l, z_{i j}}} \frac{d\left(l_{i j m}\right)}{v\left(l_{i j m}\right)}}{h_{0}}\right] \\
& \times\left\{1-\exp \left[-\frac{d\left(l_{i j k}\right)}{v\left(l_{i j k}\right) h_{0}}\right]\right\} .
\end{aligned}
$$

Consider vehicles moving along $r_{i}$. Consider calls which are generated in $z_{i j}$ by subscribers on these vehicles. Suppose that these calls are not blocked in $z_{i j} . h_{n c}\left(r_{i}, z_{i j}\right)$ is the mean channel occupancy time of these calls in $z_{i j}$. From (12) and (18), $h_{n c}\left(r_{i}, z_{i j}\right)$ is as follows:

$h_{n c}\left(r_{i}, z_{i j}\right)=\sum_{k=1}^{n_{l, z_{i j}}} h_{n c}^{\prime}\left(r_{i}, z_{i j}, l_{i j k}\right) P_{l}\left(r_{i}, z_{i j}, l_{i j k}\right)$ 


$$
\begin{aligned}
= & h_{0}-\frac{h_{0}^{2}}{\sum_{m=1}^{n_{l, z_{i j}}} \frac{d\left(l_{i j m}\right)}{v\left(l_{i j m}\right)}} \sum_{k=1}^{n_{l, z_{i j}}} \exp \left[-\frac{\sum_{m=k+1}^{n_{l, z_{i j}}} \frac{d\left(l_{i j m}\right)}{v\left(l_{i j m}\right)}}{h_{0}}\right] \\
& \times\left\{1-\exp \left[-\frac{d\left(l_{i j k}\right)}{v\left(l_{i j k}\right) h_{0}}\right]\right\},
\end{aligned}
$$

where $j<n_{z, r_{i}}$.

Next, we consider $h_{h c}\left(r_{i}, z_{i j}\right)$. Consider hand-off calls moving along $r_{i}$. Suppose that these hand-off calls enter $z_{i j}$ and are not blocked in $z_{i j}$. The mean value of the channel occupancy times of such hand-off calls is $h_{h c}\left(r_{i}, z_{i j}\right)$. In this case, we can consider that all of the hand-off calls originate at the cell boundary between $z_{i j-1}$ and $z_{i j}$; therefore, the travel time required to arrive at the cell boundary between $z_{i j}$ and $z_{i j+1}$ is equal to $\sum_{m=1}^{n_{l, z_{i j}}} \frac{d\left(l_{i j m}\right)}{v\left(l_{i j m}\right)}$. The holding time has the memoryless property. Then, in the same way as the derivation of (17),

$h_{h c}\left(r_{i}, z_{i j}\right)=h_{0}\left\{1-\exp \left[-\frac{\sum_{m=1}^{n_{l, z_{i j}}} \frac{d\left(l_{i j m}\right)}{v\left(l_{i j m}\right)}}{h_{0}}\right]\right\}$,

where $j<n_{z, r_{i}}$.

From the same reason discussed in the derivation of the hand-off probability and (A9), $h_{n c}\left(r_{i}, z_{i n_{z, r_{i}}}\right)$ and $h_{h c}\left(r_{i}, z_{i n_{z, r_{i}}}\right)$ are as follows:

$h_{n c}\left(r_{i}, z_{i n_{z, r_{i}}}\right)=h_{0}$

$h_{h c}\left(r_{i}, z_{i n_{z, r_{i}}}\right)=h_{0}$.

By using (19)-(22), we can calculate $h_{n c}\left(r_{i}, z\right)$ and $h_{h c}\left(r_{i}, z\right)$ in (2) when $z=z_{i j}$. By using (1)-(4), (13)-(16) and (19)-(22), we can define nonlinear simultaneous equations where variables are only blocking probabilities in cells. By solving the equations, we can obtain the blocking probabilities in all cells.

\section{ROAD SYSTEM AND TRAFFIC FLOW}

In the previous section, it is assumed that $Q\left(r_{i}\right), d\left(l_{i j k}\right)$ and $v\left(l_{i j k}\right)$ are given. In the practical case, we must estimate $Q\left(r_{i}\right), d\left(l_{i j k}\right)$ and $v\left(l_{i j k}\right)$. For this purpose, we utilize a method used in the transportation engineering. Traffic flow on a road system is surveyed in the field, and the results of the survey are usually represented by $O-D$ tables (Origin-Destination tables). For simplicity, this paper considers only one transportation mode. In this survey, at first, the target area is divided into some zones, which are different from radio zones 
such as cells in cellular systems, and the traffic volume from a zone to another zone is measured for every pair of zones. A zone basically has a centroid. A zone sometimes has some centroids. In this case, one of the centroids denotes the origin and the destination of the zone and other centroids, which exist on the edge of the target area, represent the origins of the traffic flows from the zones out of the target area and the destinations of the traffic flows to the zones out of the target area. All centroids in the target area correspond to centroids on the road network model. Figure 3 shows an example of the target area and the zones. In this example, the target area consists of 3 zones, and each zone has a centroid, and 7 centroids are on the edge of the target area. The element in the $i$ th row, $j$ th column in the $O-D$ table is the traffic volume from centroid $i$ to centroid $j$.

From the $O-D$ table, we can obtain the number of the vehicles moving from a centroid to another centroid per unit time; however, we do not know which routes these vehicles move along toward the destination. As described in Section 2, to analyze the mobile communication traffic characteristics, we need $Q\left(r_{i}\right)$ for all $i$. The incremental assignment method is popular and practical to estimate $Q\left(r_{i}\right)$ (Takeuchi,1986). Before we explain the incremental assignment method, we must explain the link evaluation value. Consider $r_{i}$. Let $e\left(l_{i j k}\right)$ be the link evaluation value of $l_{i j k} . e\left(l_{i j k}\right)$ is given as follows:

$e\left(l_{i j k}\right)=\frac{d\left(l_{i j k}\right)}{v\left(l_{i j k}\right)}$.

$d\left(l_{i j k}\right)$ is measured in advance. $v\left(l_{i j k}\right)$ is defined by $Q-V$ curve, which represents relation between the traffic volume and the velocity of vehicles on a link. The $Q-V$ curve is given in advance. Suppose that link $l$ connects node $a$ and node $b$. Consider all routes which include link $l$ and are directed from node $a$ to node $b$. Consider vehicles on these routes. The $Q-V$ curve for link $l$ gives the velocity of these vehicles on link $l$ from the total amount of the traffic volumes on these routes. If link $l$ is $l_{i j k}$, this velocity corresponds to $v\left(l_{i j k}\right)$. Then, the link evaluation value is also obtained.

We explain the incremental assignment method. The traffic volume from centroid $i$ to centroid $j$, which is denoted by $A_{i j}$, is given by the $O-D$ table. The incremental assignment method approximately estimates the amount of the traffic volume flowing along each route from centroid $i$ to centroid $j$. For the estimation, this method divides $A_{i j}$ into $m$ parts, and generates $m O-D$ tables, where the element in the $i$ th row, $j$ th column in each of the $O-D$ tables is equal to $A_{i j} / m$. Using the incremental assignment method, we assume the user optimal rule. Under the user optimal rule, it is assumed that each subscriber always selects the shortest route to the destination. Define that the length of $r_{i}$ is $\sum_{j=1}^{n_{z, r_{i}}} \sum_{k=1}^{n_{l, z_{i j}}} e\left(l_{i j k}\right)$. Based on the user optimal rule, the in- 
Table 1 O-D table

\begin{tabular}{lllll} 
& \multicolumn{4}{c}{ (vehicles/hour) } \\
\hline & $A$ & $B$ & $C$ & $D$ \\
\hline$A$ & 0 & 600 & 500 & 500 \\
$B$ & 700 & 0 & 0 & 400 \\
$C$ & 400 & 0 & 0 & 700 \\
$D$ & 500 & 500 & 600 & 0 \\
\hline
\end{tabular}

cremental assignment method assigns each of the divided traffic volumes to the shortest route as follows: First, the incremental assignment method calculates the shortest route between each pair of centroids under the condition that there exists no vehicle on the network; and secondly, the incremental assignment method assigns $A_{i j} / m$ to the shortest route between centroid $i$ and centroid $j$ for all $i, j$. After these assignments, the traffic volume on each route changes, and the link evaluation value of each link also changes. Then, the shortest route between each pair of centroids also changes. Hence, in the next step, the incremental assignment method calculates the shortest route between each pair of centroids again, and assigns $A_{i j} / m$ to the new shortest route. After repeating this procedure $m$ times, the traffic volume $A_{i j}$ is all assigned to routes from centroid $i$ to centroid $j$. Consequently, $Q(r)$ is obtained for all available routes.

Let us consider an example shown in Figure 1. In this network, there are four centroids denoted by $A, B, C$ and $D$. In this example, there are thirteen links which are numbered. Suppose that the length of link 1 is $1000 \sqrt{3} \mathrm{~m}$ and the length of each of the other links is $500 \sqrt{3} \mathrm{~m}$. The cell radius is $1,000 \mathrm{~m}$. An example of the $O-D$ table is shown in Table 1. $Q-V$ curves are represented in Figure 4. In this figure, $Q_{\max }$ is the largest traffic volume. We assume that the total amount of the traffic volumes in one direction on a link is smaller than $Q_{\max }$. From the $O-D$ table and the $Q-V$ curve, the incremental assignment method gives the traffic assignment results as shown in Table 2. From Table 1 , from centroid $C$ to centroid $D, 700$ vehicles move per hour. From Table 2,140 vehicles and 560 vehicles move along route 7 and route 11 per hour, respectively. This is also explained in Figure 5. Also, from the traffic volumes on routes $3,5,7$ and 11 , the traffic volume flowing toward centroid $D$ on link 9 is 1600 vehicles per hour in total. 


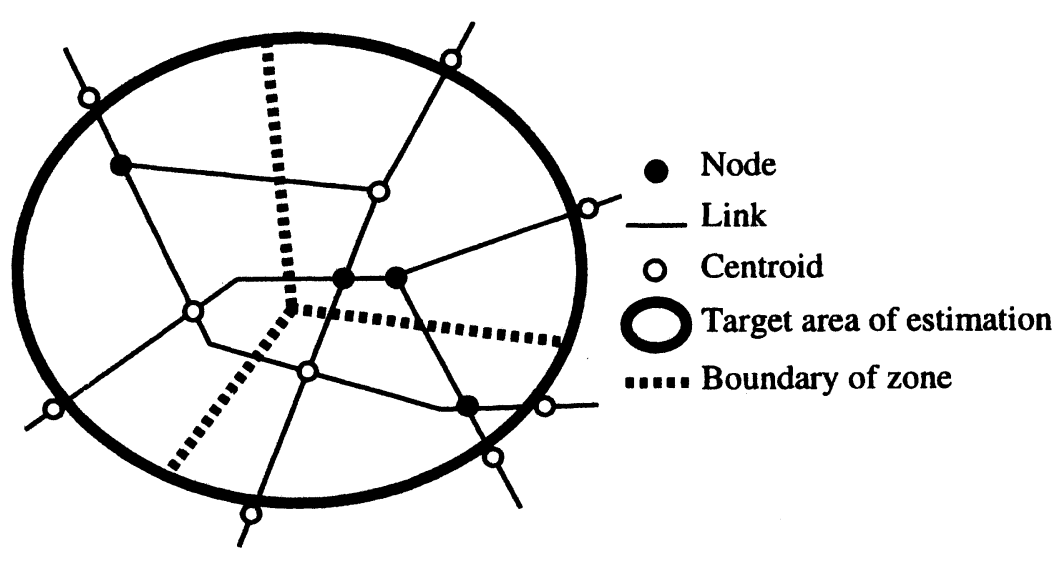

Figure 3 Target area and zones.

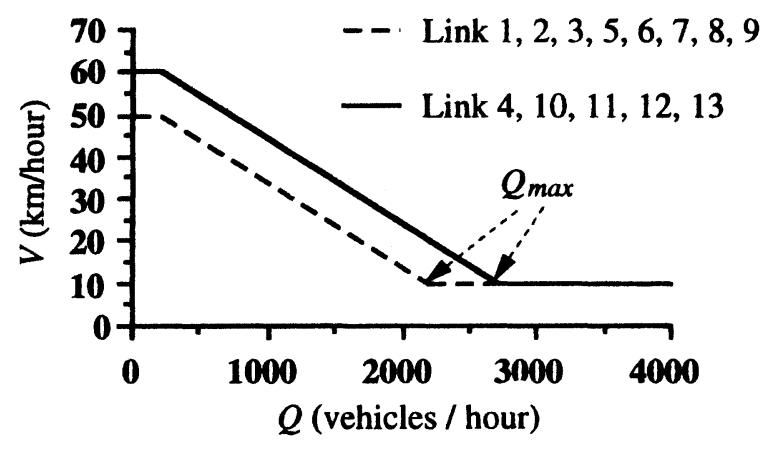

Figure 4 Q-V curve.

\section{NUMERICAL RESULTS}

We give numerical results of the analysis technique. We analyze the example described in Section 3. The following is assumed: $S=3, \lambda=0.01$ and $h_{0}$ is 1.5 minutes.

Table 3 shows the numerical results and the computer simulation results on the same model. From Table 3, it seems that the numerical results of the analysis agree well with the computer simulation results. In the computer simulation, the distribution of hand-off call arrivals is not given. On the other 
Table 2 Estimation results

\begin{tabular}{lll}
\hline Route & Links & Traffic volume (vehicles/hour) \\
\hline 1 & 5,1 & 600 \\
2 & $6,3,2$ & 500 \\
3 & $7,8,9$ & 500 \\
4 & 1,5 & 700 \\
5 & $1,5,7,8,9$ & 400 \\
6 & $2,3,6$ & 400 \\
7 & $2,3,6,7,8,9$ & 140 \\
8 & $9,8,7$ & 500 \\
9 & $9,8,7,5,1$ & 500 \\
10 & $9,8,7,6,3,2$ & 120 \\
11 & $2,4,11,12,13,10,9$ & 560 \\
12 & $9,10,13,12,11,4,2$ & 480 \\
\hline
\end{tabular}

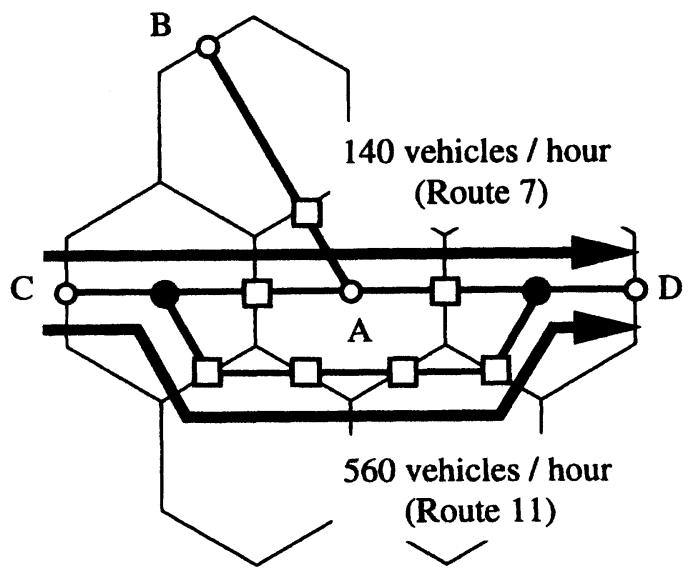

Figure 5 Traffic volumes on two routes from centroid $C$ to centroid $D$.

hand, the numerical results are obtained by assuming that the distribution of hand-off call arrivals is the Poisson distribution. From the agreement, the validity of this assumption is confirmed. 
Table 3 Blocking probability

\begin{tabular}{lll}
\hline Cell & Theory & Simulation \\
\hline 1 & 0.100267 & 0.100281 \\
2 & 0.076861 & 0.066659 \\
3 & 0.257632 & 0.257675 \\
4 & 0.252321 & 0.252492 \\
5 & 0.001099 & 0.001050 \\
6 & 0.000990 & 0.000798 \\
\hline
\end{tabular}

\section{CONCLUSIONS}

We have proposed a technique to analyze the communication traffic characteristics of the 2-dimensional cellular systems on a road systems model. By using this technique, we can estimate the communication characteristics from the traffic flow on the road system measured in the field.

As discussed in this paper, the results of the transportation engineering field can be applied to the teletraffic engineering for mobile communications. We may use other results in the transportation engineering field for further development of mobile communication systems. Establishment of the new engineering field by considering relation between the transportation engineering and the teletraffic engineering is our future problem.

\section{ACKNOWLEDGMENT}

A part of this work was conducted with the Grants for International Collaborative Research provided by the Telecommunications Advancement Organization of Japan (TAO).

\section{REFERENCES}

Cooper, R. B. (1981) Introduction to queuing theory. Elsevier North Holland, Inc.

Guerin, R. A. (1987) Channel occupancy time distribution in a cellular radio system. IEEE Transactions on vehicular technology, Vol. VT-35, No. 3, 89-99.

Hong, D. and Rappaport, S. S. (1986) Traffic model and performance analysis for cellular mobile radio telephone systems with prioritized and nonprioritized handoff procedures. IEEE Transactions on vehicular technology, Vol. VT-35, No. 3, 77-92. 
Inoue, M., Morikawa, H. and Mizumachi, M. (1994) Optimum system architecture of microcellular mobile communications. Technical report of IEICE, RCS94-34, 43-8 (in Japanese).

Nanda, S. (1993) Teletraffic models for urban and suburban microcells: cell sizes and handoff rates. IEEE Transactions on vehicular technology, Vol. 42, No. 4, 673-82.

Otsuka, A., Sengoku, M., Yamaguchi, Y. and Abe, T. (1987) Basic study on mobile flows and mobile telephone traffic. Technical report of IEICE, CAS86-249, 81-8 (in Japanese).

Rappaport, S. S. (1991) The multiple-call hand-off problem in high-capacity cellular communications systems. IEEE Transactions on vehicular technology, Vol. 40, No. 3, 546-57.

Rappaport, S. S. and Hu, L. R. (1994) Microcellular communication systems with hierarchical macrocell overlays: traffic performance models and analysis. Proceedings of the IEEE, Vol. 82, No. 9, 1383-97.

Takeuchi, D., Honda, Y. and Aoshima, N. (1986) Transportation engineering. Kashima Syuppankai (in Japanese).

\section{BIOGRAPHY}

Keisuke Nakano was born in Niigata, Japan in 1966. He received the Ph.D. degree from Niigata University in 1994. He is currently a Research Associate of Niigata University. His research interests include performance analysis of communication networks and mobile communication systems. He received the Best Paper Award of the 1995 IEEE International Conference on Neural Networks and Signal Processing. He also received the Best Paper Award from IEICE in 1997. He is a member of IEICE, IEEE and ACM.

Katsushi Saita was born in Niigata, Japan in 1973. He received the B.E. degree from Niigata University in 1996. He is currently a M.E. candidate of Niigata University. His research interests include performance analysis of communication networks. He is a member of IEICE.

Masakazu Sengoku was born in Nagano prefecture, Japan in 1944. He received the B.E. degree from Niigata University, Niigata, Japan, in 1967 and the M.E. and Ph.D. degrees from Hokkaido University in 1969 and 1972, respectively. In 1972, he joined the staff at Department of Electronic Engineering, Hokkaido University as a Research Associate. In 1978, he was an Associate Professor at Department of Information Engineering, Niigata University, where he is presently a Professor. His research interests include network theory, graph theory, transmission of information and mobile communications. He received the Paper Awards from IEICE in 1992, 1996 and 
1997, respectively. And he received the Best Paper Award of the 1995 IEEE International Conference on Neural Networks and Signal Processing. He is a member of IEICE, IEEE and IPS of Japan.

Yoshihide Yamada was born in Wakayama, Japan in 1948. He received the B.S. and M.S. degrees from Nagoya Institute of Technology in 1971 and 1973, respectively. And he received the D.E. degree from Tokyo Institute of Technology in 1989. In 1973, he joined the Electrical Communication Laboratories of NTT, Japan. Till 1984, he was engaged in research and development of reflector antennas for terrestrial and satellite communications. From 1985, he has been engaged in R\&D of base station antennas for mobile radio systems. In 1993, he moved to NTT Mobile Communications Network Inc. He conducted the same subject as an executive research engineer. In 1995, he was temporarily transferred to YRP Mobile Telecommunications Key Technology Research Laboratories Co., Ltd. He is a Head of the Communication Systems Department. At the same time, he has been a guest professor of the cooperative research center of Niigata University, and a Lecturer of Science University of Tokyo, both from 1996. He is a member of IEICE and IEEE.

Shoji Shinoda was born on December 15, 1941, in Hokkaido, Japan. He received the B.E., M.E. and D.E. degrees, all in electrical engineering, from Chuo University, Tokyo, Japan, in 1964, 1966 and 1973, respectively. In April, 1965, he joined the Faculty of Science and Engineering, Chuo University, Tokyo, Japan. Since then, he has engaged in education and research in the fields of electrical circuit theory, network flow and tension theory, discrete systems, and mobile communication systems. He is now a Professor of the Department of Electrical and Electronic Engineering and the Dean of the Graduate School of Science and Engineering, Chuo University. He has published more than one hundred technical papers in the aforesaid fields. He is also the recipient of the 1992 IEICE Best Paper Award, the 1997 IEICE Best Paper Award and the Best Paper Award of the 1995 IEEE International Conference on Neural Networks and Signal Processing. He is now a member of IEICE, IEEE, SICE (Society of Instrument and Control Engineers), JSST (Japan Society of Simulation Technology) and KITE (Korean Institute of Telematics and Electronics). 